\title{
Usefulness of semi-quantification of ischemic myocardium after adenosine stress magnetic resonance
}

\author{
Lorenzo Monti ${ }^{1,2^{*}}$, Marco Tramarin ${ }^{2}$, Margherita Calcagnino ${ }^{1}$, Veronica Lisignoli ${ }^{1}$, Barbara Nardi ${ }^{1}$, Luca Balzarini $^{2}$ \\ From 16th Annual SCMR Scientific Sessions \\ San Francisco, CA, USA. 31 January - 3 February 2013
}

\section{Background}

Adenosine stress MR shows high sensitivity and intermediate-to-high specificity; therefore, there is a number of false positive patients that is referred to useless coronary angiography studies after a positive adenosine stress MR. We sought to verify whether the (semi) quantification of the amount of ischemic myocardium can improve the management of patients after a positive adenosine stress MR study. According to previous nuclear medicine evidence, we defined a $10 \%$ LV mass cut-off for the diagnosis of clinically relevant ischemia.

\section{Methods}

80 MR studies were classified as positive or negative according to the presence or absence of a reversible perfusion defect ("old approach"). We re-analyzed the perfusion studies dividing each one of the 16 AHA segment of the 3 perfusion slices in 2 similar sub-segments ( subepicardial and subendocardial): each one of the resulting 32 segment represent about $3 \%$ of the myocardial mass. We re-defined ("new approach") as ischemic the patients with $>3$ subsegments ( $>10 \%$ of LV mass) with reversible lesions, and as non-ischemic the patients with 3 or less positive subsegments. Patients were classified as affected by clinically relevant ischemia if the post-adenosineMR coronary angiography confirmed coronary lesions $>50 \%$, and non-affected if the angio was negative and/or no MACE were observed after a $>6$ months follow-up.

\section{Results}

Mean age 60.7 years old. Mean follow-up $=18$ months (5-37). Pre-test prevalence of CHD: 64\%. Prevalence of clinically relevant ischemia $=17.5 \%$. With the old approach we obtained a sensitivity and specificity of $92.9 \%$ and 72.7 respectively. With the new approach we obtained a sensitivity and specificity of $85.7 \%$ and $90.9 \%$ respectively. Global accuracy of the stress MR exam increased from 76.2 to $90 \%$.

\section{Conclusions}

A visual semi-quantification of the ischemic myocardium improves patient management after a positive adenosine stress MR study.

\section{Funding}

None.

\section{Author details}

${ }^{1}$ Cardiology, I.R.C.C.S. Istituto Clinico Humanitas, Rozzano(MI), Italy. ${ }^{2}$ Radiology, I.R.C.C.S. Istituto Clinico Humanitas, Rozzano (MI), Italy.

Published: 30 January 2013

doi:10.1186/1532-429X-15-S1-E58

Cite this article as: Monti et al: Usefulness of semi-quantification of ischemic myocardium after adenosine stress magnetic resonance. Journal of Cardiovascular Magnetic Resonance 2013 15(Suppl 1):E58.

${ }^{1}$ Cardiology, I.R.C.C.S. Istituto Clinico Humanitas, Rozzano(MI), Italy

Full list of author information is available at the end of the article

(c) 2013 Monti et al; licensee BioMed Central Ltd. This is an Open Access article distributed under the terms of the Creative Commons 